\title{
The complex relationship between infertility and psychological distress (Review)
}

\author{
GABRIELA SIMIONESCU $^{1-3}$, BOGDAN DOROFTEI $^{1,2}$, RADU MAFTEI $^{2-4}$, BIANCA-ELENA OBREJA $^{5}$, \\ EMIL ANTON $^{1,2}$, DELIA GRAB ${ }^{1,2}$, CIPRIAN ILEA $^{1,2}$ and CARMEN ANTON ${ }^{1,6}$ \\ ${ }^{1}$ Department of Mother and Child Medicine, University of Medicine and Pharmacy 'Gr. T. Popa' Iasi, Faculty of Medicine, \\ 700115 Iasi; ${ }^{2}$ Clinical Department, Clinical Hospital of Obstetrics and Gynecology 'Cuza Voda', 700038 Iasi; \\ ${ }^{3}$ Origyn Fertility Center, 700032 Iasi; ${ }^{4}$ Department of Morphostructural Sciences, University of Medicine and Pharmacy \\ 'Gr. T. Popa' Iasi, Faculty of Medicine, 700115 Iasi; ${ }^{5}$ Faculty of Letters, Alexandru Ioan Cuza University, \\ 700506 Iasi; ${ }^{6}$ Clinical Department, Sf. Spiridon Clinical Hospital, 700111 Iasi, Romania
}

Received July 1, 2020; Accepted December 18, 2020

DOI: $10.3892 /$ etm.2021.9737

\begin{abstract}
Infertility is defined as the inability to procreate, or carry or deliver a baby naturally. The majority of specialists describe infertility as being unable to get pregnant after having tried for at least one year. The relationship between infertility and psychological stress is complex. On the one hand, infertile couples are subject to greater stress and have a greater risk of developing psychological disorders compared with normal, healthy couples. On the other hand, high levels of psychological distress have been indicated to increase infertility. Therefore, in the present review, the main factors that may lead to increased stress in couples who try to conceive, psychological stress as the reason for infertility, and the therapies that can help decrease psychological distress and increase chances of pregnancy are underlined. In addition to the psychological side effects that may occur from infertility itself, a range of other side effects can be caused by hormones and drugs used to treat infertility. Additionally, problem during erection and ejaculation can cause of psychological distress, which can lead to infertility among men. Psychotherapy is the main intervention recommended for couples who suffer from any form of infertility. Ideally, counselling should begin before patients start any medical intervention to help with their infertility.
\end{abstract}

Correspondence to: Dr Emil Anton, Department of Mother and Child Medicine, University of Medicine and Pharmacy 'Gr. T. Popa' Iasi, Faculty of Medicine, 11 Universitatii Street, 700115 Iasi, Romania

E-mail: emil.anton@yahoo.com

Key words: infertility, psychological distress, anxiety, depression, pregnancy

\section{Contents}

1. Introduction

2. Stress of infertility

3. Psychological factors as the reason for infertility

4. Correlation between psychological disorders and infertility

5. Therapies that may help

6. Conclusion

\section{Introduction}

Infertility is defined as the inability to procreate, carry or deliver a baby naturally (1). The majority of specialists describe infertility as being unable to get pregnant after having tried for at least one year (1). Women who can get pregnant but have recurrent miscarriages are also considered to be infertile. The World Health Organization's definition of infertility is referred to as following a period of 24 months of trying to conceive (2).

Infertility affects an estimated $10-15 \%$ of reproductive age couples worldwide $(1,2)$. The percentage of couples seeking infertility treatment has risen dramatically in recent years as a result of factors such as postponement of childbearing in women, expansion of newer and more successful techniques for infertility treatment and increased publicity of these available therapies (3).

The relationship between infertility and psychological stress is complex. On the one hand, a previous study (4) indicated that infertile couples are subject to greater stress and have an increased risk of developing psychological disorders compared with healthy couples. On the other hand, high levels of psychological distress have been revealed to increase infertility (5). Therefore, in the present review, the main factors that may lead to increased stress in couples who try to conceive, psychological stress as the reason for infertility and the therapies that can help decrease psychological distress and increase pregnancy chances are discussed.

\section{Stress of infertility}

Psychological stress. Infertility can cause psychologically distress, emotionally stress and financial difficulties for both 
partners (3). Typical reactions to infertility include shock, sadness, depression, anger and frustration, loss of self-esteem and self-confidence and a general loss of sense of control (1).

Although infertility is not a life-threatening issue, it is still considered a stressful life experience for couples. The high stress of infertility might be attributed to the fact having a child is considered to be important in general society (2).

Furthermore, infertile couples often conceal their emotions, ideas and beliefs because infertility is still considered to be a private subject. Therefore, infertile couples may be exposed to social pressure. In addition, an individual relationship with their partner as well as that with friends and family members may suffer. These family members or friends may provide meaningful views and suggestions that may cause further distress. Therefore, couples with infertility problems may reduce social interaction, especially with pregnant women and friends who have children (3).

Additionally, infertility may negatively impair an individuals' self-esteem. For example, a study in a Jordanian population aimed to examine the effects of experiences of rural women on infertility (6). In the Jordanian rural sociocultural context, this aforementioned study aimed to investigate reactions to infertility and its implications. The results indicated that female reactions to infertility included submission and docility, self-isolation and internalisation of anger and sadness. In addition, women with infertility were revealed to have a negative perception about themselves and their sexuality (6).

Psychological stress is also perceived as a potential clinical risk factor that may reduce male fertility (7-9). Studies investigating the correlation between male infertility and psychological stress have reported conflicting results (7-12). Some authors have succeeded in demonstrating that an inverse relationship exists between psychological stress and semen parameters (7-11). However, a previous study (12) has reported results that failed to demonstrate this relationship.

More specifically, there are studies that have identified alterations in a number of different sperm parameters in diverse, stressful circumstances, such as in students during exam periods $(7)$, times of war $(8,9)$, periods of elevated stress in their professional career (10) or during mourning (11). However, a study by Hjollund et al (12) contradicted the aforementioned results by indicated that an individual's everyday psychological stress has little effect on semen quality. Moreover, treatment for reducing infertility itself can become a factor that increases stress in men (13). A previous study demonstrated that men from couples who fail to achieve pregnancy despite treatment will experience negative feelings such as frustration and disappointment caused mainly by prolonged efforts in terms of taking medication and expensive treatment costs (13). Furthermore, fertility problems, such as alterations in semen parameters, can lead to other stress-related conditions such as erectile dysfunction and premature and retrograde ejaculation, which can further negatively influence fertility status (9).

Side effects from medication. In addition to the psychological side effects that may occur from infertility itself, a range of other side effects can be caused by hormones and drugs used to treat infertility. For example, the synthetic oestrogen clomiphene citrate (Clomid, Serophene), which is often prescribed as it improves ovulation and increases sperm production, can trigger anxiety, interruptions of sleep and irritability in women (11). It is important to mention that these side effects have not been reported in men (13). Other medications for infertility may cause issues such as depression, mania, irritability and difficulty in concentrating (14). For example, letrozole, which is an oestrogen receptor blocker, may cause various side effects such as severe headaches, dizziness, fatigue, sleeplessness, breast pain or an abnormal cycle (14). Lupron, which is another drug commonly used in the treatment of infertility, has been indicated to cause mood swings, depression and anxiety symptoms, vaginal dryness, acne, joint pain and decreased sex drive in some women (15). As aforementioned, infertility itself may lead to various psychological side effects. It may be difficult for patients and clinicians to understand which responses are psychological and which are caused by medication (14). Therefore, a classification of these possible side effects is vital (Table I indicates the possible negative side effects of the most popular treatments used for infertility) (14-16).

Financial difficulties. Few countries worldwide offer insurance coverage for the treatment of infertility (1). The coverage range of the treatment varies, and it is not $100 \%$ effective. Therefore, the cost of infertility treatment is very high. For example, the average cost of an in vitro fertilisation (IVF) cycle using fresh embryos is $\$ 8,158$, with fertility drugs adding $\$ 3,000$ to $\$ 5,000$ per cycle (17).

Failure to acquire specialised treatment can lead to feelings of helplessness and desperation for couples who do not have insurance coverage or the means to pay for the therapy (17). Even patients with insurance coverage may discover that they have to large sums of money with co-payments or may experience coverage constraints (17).

Probability and outcomes. Infertility procedures help only $\sim 50 \%$ of patients to become parents, with this probability of achieving pregnancy lowering with age (2). Patients who undergo successful IVF procedures may be overjoyed, but new types of emotional and psychological stress are also experienced during pregnancy and after birth. For instance, women who have endured numerous miscarriages can be anxious if they can carry to term. Older couples may discuss whether prenatal tests such as amniocentesis should be undertaken, as this is a common source of stress (18).

\section{Psychological factors as the reason for infertility}

Women. It has previously been identified that causes of infertility among men and women are widespread $(3,6,16)$. Causal factors for infertility are not limited to medical factors but extend to psychological variables (19). Women's emotional stress can be indicated as tubal spasms, anovulations, and vaginism (1). In addition, during ovulation, women can unintentionally avoid sexual intercourse (2). Women with infertility have a number of psychological commonalities. Although the majority of women appear to enjoy getting pregnant and verbally expressing their desire, they may conceal adverse opinions and fear of pregnancy (20). These fears may be caused by pregnancy, delivery or maternity. Changing body shape 
Table I. The most commonly used fertility drugs, their use and the common observed side effects.

\begin{tabular}{lll}
\hline Fertility drug $\quad$ Use & Possible side effects
\end{tabular}

Clomid

Clomid works by blocking the receptors in the body that react to the estrogen hormone, leading to an increase in circulating estrogen

Letrozole

Lupron and GnRH agonist side

Oral contraceptives
Used off-label as a fertility drug. (Letrozole, also known as Femara, is intended for use in the treatment of breast cancer). Letrozole works in a similar way as Clomid (blocking estrogen receptors). Studies have found that women with PCOS and women who are Clomid resistant (do not ovulate on Clomid) may have more success with Letrozole (52)

Gonadotropin fertility drugs are injectable hormones. They include drugs like Gonal-F (FSH), Follistim and Ovidrel (hCG). Injectables may be used alone or in combination with other drugs during an IVF (in vitro fertilization) cycle

GnRH agonists like Lupron are most commonly used during IVF treatment. They shut down the body's natural reproductive system so that the physician can control ovarian stimulation and maturation. Low estrogen levels are responsible for many of the side effects of GnRH agonists.

Used in the treatment of infertility, but they often used in IVF treatment
Bloating and abdominal discomfort, weight gain, headaches, mood swings, nausea, dizziness, breast tenderness, abnormal menstrual bleeding/spotting, vaginal dryness, blurred vision

Fatigue, dizziness, headache, bloating/abdominal discomfort, hot flashes, blurred vision, trouble sleeping, abnormal menstrual bleeding/spotting, breast pain, vision disturbances

Headaches, nausea/upset stomach, upper respiratory tract infection, bloating/abdominal tenderness, mood swings, acne, weight gain, abnormal menstrual bleeding/spotting, injection site soreness and redness, dizziness

Headache, mood swings/depression/anxiety, vaginal dryness, acne, general body aches/joint pain, nausea, fluid retention, upset stomach, weight gain, decreased sex drive, dizziness, injection site soreness

Depression, weight gain

IVF, in vitro fertilisation; GnRH, gonadotropin-releasing hormone; hCG, human chorionic gonadotropin; FSH, follicle-stimulating hormone; PCOS, polycystic ovary syndrome.

during pregnancy, fear of losing one's life or the child during delivery, or fear of failing as a mother are some of the potential underlying causes of stress (21). A previous study (22) have demonstrated that a more affectionate and unrestricted bond could be created between partners if women were encouraged to communicate feelings, which could subsequently lead to increased chances of pregnancy.

Men. In contrast, impotence with erection and ejaculation can be the root cause of psychological issues, which can lead to infertility among men (1). These issues may arise from birth or may arise later in life. Furthermore, temporary impotence can be experienced at any point in life in the vast majority of men. Most often, impotence is caused by psychological triggers and specifically, previous psychological traumas, eating disorders and overprotective and over-affectionate mothers have been indicated to be among the main psychological variables that may lead to impotence $(23,24)$.

\section{Correlation between psychological disorders and infer- tility}

A number of studies have explored the connection between infertility and a variety of psychological disorders. In a study on 112 females with infertility, some types of psychiatric disorders (for example, anxiety and depression) were indicated to be present in $40 \%$ of the participants (25). Furthermore, the most prevalent diagnoses in women with infertility in Japan was anxiety disorder (23\%), mild depressive disorder (17\%) and dysthymic disorder (9.8\%) (25). In addition, the frequency of psychiatric disorders has been demonstrated to be significantly higher in individuals with a diagnosed infertility problem compared with healthy counterparts (6). These widespread psychological disorders in individuals with infertility-related problems are not explained by other cultural features of Japanese society (25). For example, in an 11,000-person study conducted in an American community, generalised anxiety disorder was also found to be significantly associated with infertility (26).

Furthermore, a high incidence of anxiety and depression has been identified in patients with infertility in a number of studies from distinct societies (27). For example, the prevalence of anxiety among the infertile group was higher compared with patients diagnosed with HIV-positive, cancer, heart diseases, or other serious, life-threatening chronic diseases (27). In another relevant study, mood disorders were reported to be 3.4 times higher and anxiety disorder 2.7 times 
more widespread in the infertile group of patients compared with the control group (28). Other common psychiatric problems among patients with infertility include disorders of sexual function, somatisation, dysthymia, frequent panic attacks, obsessive-compulsive disorder and social anxiety disorder $(1,25)$. Furthermore, infertility has been associated with eating disorders such as anorexia nervosa, bulimia nervosa and obesity (29).

In addition, previous studies have indicated that alcohol and drug addiction are prevalent among individuals with infertility (26). Furthermore, some studies have indicated that women exhibit increased emotional stress due to infertility (30). It has also been noted that hormonal imbalance in the hypothalamic hypophyseal ovarian axis or hormonal medicines could also lead to mood disorders among patients undergoing infertility treatment (31). In a study that examined mental state and personality profile in patients who are infertile, at least one mental disorder was identified in $83.8 \%$ of women. Furthermore, $52 \%$ of the participants exhibited mild or serious personality disorders (32). In the same study, it was also disclosed that there was a greater level of depression and anxiety in the group of patients who had infertility and a lower level of mental composure linked to personality traits.

Gender differences. Some differences in traits and disorders have been previously associated with infertility (26). It has also been demonstrated that women with infertility present greater levels of depression compared with infertile men (33). Specifically, depression is reported to be more prevalent among women with infertility, while there is a greater amount of psychosomatic distress among men with infertility (34). In addition, a greater level of experienced stress and a deteriorated quality of life has been indicated among infertile men with elevated alexithymia characteristics (35). On the other hand, women with infertility have revealed cognitive dysfunction and increased anxiety and depression $(36,37)$. Previous studies have indicated that men with infertility score higher on the scales of relationship satisfaction compared with women (38). A possible explanation for these results may be that women feel more responsible and guilty than men during the process of trying to concieve. Furthermore, women with infertility are exposed to greater social pressure and stigma $(39,40)$.

Variables that may mediate the correlation between psychological disorders and infertility. It has been previously demonstrated that insufficient psychological assistance, ineffective therapy, low socioeconomic status and lack of partner assistance are associated with increased chances of developing depression (40). For example, a study showed that an individuals partner and relatives adverse responses regarding infertility was associated with higher levels of anxiety and depression and reduced self-esteem (36).

Regarding other psychological variables that may be associated with infertility, it has been reported that unexplained infertility is associated not only with elevated levels of anxiety but also with high levels of stress (41). Additionally, genetic infertility is indicated to be more associated with depression (42).

Regarding the main factors that are associated with male infertility, stress levels are demonstrated to have a higher impact on male-related infertility issues. For example, in a study, couples were asked to avoid expressing negative feelings regarding male-caused infertility issues. The results of the aforementioned study indicated that not expressing negative feelings regarding men's infertility was positively associated with increased pregnancy ratios (43).

Furthermore, a previous study stated that the period of infertility was more dependent on depression level rather than the duration of treatment (36). These results and associations are important because at the beginning of the therapy, couples may assume they will conceive quickly, and as the duration of infertility increase, depression, anxiety or other psychological disorders may increase. Therefore, in the subsequent section of the present study, the recommended treatments that couples suffering from any type of infertility-related problem could follow are outlined.

\section{Therapies that may help}

Psychotherapy. Psychotherapy is an important intervention that should be recommended for couples suffering from any form of infertility. Therefore, counselling should ideally begin before patients start any medical intervention to help with infertility (44). As some studies have indicated, addressing psychological problems such as depression, anxiety and stress may help increase the chance of conception (44). In addition, studies have indicated that interpersonal treatment, which focuses on enhancing relationships or resolving disputes with others, and cognitive behavioural therapy, can help patients with infertility with depression and anxiety $(45,46)$. Studies have revealed that psychotherapy can be separately provided with great results to couples or in a community $(47,48)$.

Furthermore, psychotherapy helps boost coping strategies or assist in decision-making during treatment. Additionally, therapy may help patients with various mood or sleep disorders.

Relaxation techniques. In addition to the psychological help that couples who cannot conceive a baby should receive, other complementary interventions should also be advocated. Given that infertility and its therapy often cause significant stress, multiple relaxation techniques are recommended by specialists: Meditation, deep breathing, guided imagery and yoga.

Previous studies have emphasised the effect of yoga on decreasing psychological stress among individuals undergoing infertility treatment (49-51). Specifically, a study demonstrated that yoga intervention increased the quality of life and decreased negative feelings and thoughts that were associated with infertility (50). The aforementioned study revealed that participating in yoga classes also decreased symptoms of anxiety and depression in women undergoing IVF (50). Furthermore, the results of previous studies have demonstrated that structured yoga classes lasting six weeks decreased anxiety by $20 \%$ and anxiety scores by $12 \%$ (52).

\section{Conclusion}

The diagnosis of infertility can be an overwhelming burden for couples. This diagnosis may lead to major psychiatric disorders 
such as depression, anxiety and low self-esteem. Therefore, couples should be offered counselling and support as they undergo infertility treatment. It has been well documented that psychological interventions for couples with infertility have the capacity to reduce symptoms of anxiety and depression and significantly increase pregnancy rates $(25,26)$.

Therefore, although the psychological effects of infertility can be significant, the majority of patients can be helped with the proper intervention to achieve some type of resolution: Whether individuals become parents to biological kids, adopt kids or decide to not have children.

\section{Acknowledgements}

Not applicable.

\section{Funding}

No funding was received.

\section{Availability of data and materials}

The datasets used and/or analyzed during the current study are available from the corresponding author on reasonable request.

\section{Authors' contributions}

GS, EA and CA provided substantial contributions to conception and design of the present study. BD, RM, BEO, DG and CI acquired the data and contributed to analysis and interpretation of data. GS and EA confirmed the authenticity of the raw data. GS, EA and CA drafted the article. All authors read and approved the final manuscript.

\section{Ethics approval and consent to participate}

Not applicable.

\section{Patient consent for publication}

Not applicable.

\section{Competing interests}

The authors declare that they have no competing interests.

\section{References}

1. Vander Borght M and Wyns C: Fertility and infertility: Definition and epidemiology. Clin Biochem 62: 2-10, 2018.

2. Deshpande PS and Gupta AS: Causes and prevalence of factors causing infertility in a public health facility. J Hum Reprod Sci 12: 287-293, 2019.

3. Boivin J, Bunting L, Collins JA and Nygren KG: International estimates of infertility prevalence and treatment seeking: Potential need and demand for infertility medical care. Hum Reprod 22: 1506-1512, 2007.

4. Greil AL: Infertility and psychological distress: A critical review of the literature. Soc Sci Med 45: 1679-1704, 1997.

5. Drosdzol A and Skrzypulec V: Quality of life and sexual functioning of Polish infertile couples. Eur J Contracept Reprod Health Care 13: 271-281, 2008.
6. Daibes MA, Safadi RR, Athamneh T, Anees IF and Constantino RE: Half a woman, half a man; that is how they make me feel: A qualitive study of rural Jordanian women's experience of infertility. Cult Health Sex 20: 516-530, 2018.

7. Eskiocak S, Gozen AS, Taskiran A, Kilic AS, Eskiocak M and Gulen S: Effect of psychological stress on the L-arginine-nitric oxide pathway and semen quality. Braz J Med Biol Res 39: 581-588, 2006.

8. Abu-Musa AA, Nassar AH, Hannoun AB and Usta IM: Effect of the Lebanese civil war on sperm parameters. Fertil Steril 88 1579-1582, 2007.

9. Zorn B, Zucur V, Stare J and Meden-Vrtovec H: Decline in sex ratio at birth after 10-day war in Slovenia: Brief communication. Hum Reprod 17: 3173-3177, 2002.

10. Jurewicz J, Hanke W, Sobala W, Merecz D and Radwan M: The effect of stress on the semen quality. Med Pr 61: 607-613, 2010.

11. Fenster L, Katz DF, Wyrobek AJ, Pieper C, Rempel DM, Oman D and Swan SH: Effect of psychological stress on human semen quality. J Androl 18: 194-202, 1997.

12. Hjollund NH, Bonde JP, Henriksen TB, Giwercman A and Olsen J; Danish First Pregnancy Planner Study Team: Job strain and male fertility. Epidemiology 15: 21-27, 2004.

13. Schneid-Kofman N and Sheiner E: Does stress affect male infertility? - a debate. Med Sci Monit 11: 11-13, 2005.

14. Choi SH, Shapiro H, Robinson GE, Irvine J, Neuman J, Rosen B, Murphy J and Stewart D: Psychological side-effects of clomiphene citrate and human menopausal gonadotrophin. J Psychosom Obstet Gynaecol 26: 93-100, 2005.

15. Warnock JK, Bundren JC and Morris DW: Depressive mood symptoms associated with ovarian suppression. Fertil Steril 74: 984-986, 2000.

16. Wilkins KM, Warnock JK and Serrano E: Depressive Symptoms related to infertility and infertility treatments. Psychiatr Clin North Am 33: 309-321, 2010.

17. Macaluso M, Wright-Schnapp TJ, Chandra A, Johnson R, Satterwhite CL, Pulver A, Berman SM, Wang RY, Farr SL and Pollack LA: A public health focus on infertility prevention, detection and management. Fertil Steril 93: 16.e1-10, 2010.

18. Rahim R and Majid S: Aetiological factors of infertility. JPMI 18: 166-171, 2004.

19. Mahlstedt PP: The psychological component of infertility. Fertil Sterili 43: 335-346, 1985.

20. Matsubayashi H, Hosaka T, Izumi S, Suzuki T and Makino T: Emotional distress of infertile women in Japan. Hum Reprod 16: 966-969, 2011.

21. Melender HL: Experiences of fears associated with pregnancy and childbirth: A study of 329 pregnant women. Birth 29: 101-111, 2002.

22. Silveira ML, Ertel KA, Dole N and Chasan-Taber L: The role of body image in prenatal and postpartum depression: A critical review of the literature. Arch Womens Ment Health 18: 409-421, 2015.

23. Collins A, Freeman EW, Boxer AS and Tureck R: Perception of infertility and treatment stress in females as compared with males entering in vitro fertilization treatment. Fertil Steril 57: 350-356, 1992.

24. Fido A and Zahid MA: Coping with infertility among Kuwaiti women: Cultural perspectives. Int J Soc Psychiatry 50: 294-300, 2004.

25. Chen TH, Chang SP, Tsai CF and Juang KD: Prevalence of depressive and anxiety disorders in an assisted reproductive technique clinic. Hum Reprod 19: 2313-2318, 2004.

26. King RB: Subfecundity and anxiety in a nationally representative sample. Social Sci Med 56: 739-751, 2003.

27. Kainz K: The role of the psychologist in the evaluation and treatment of infertility. Womens Health Issues 11: 481-485, 2011.

28. Klemetti R, Raitanen J, Sihvo S, Saarni S and Koponen P: Infertility, mental disorders and well-being: A nationwide survey. Acta Obstet Gyn Scan 89: 677-682, 2010.

29. Gulseren L, Cetinay P, Tokatlioglu B, Sarikaya OO, Gulseren S and Kurt S: Depression and anxiety levels in infertile Turkish women. J Reprod Med 51: 421-426, 2006.

30. Kirca $\mathrm{N}$ and Pasinoglu T: Psychosocial problems during infertility treatment. Curr App Psychiatry 5: 162-178, 2013.

31. De D, Roy PK and Sarkhel S: A psychological study of male, female related and unexplained infertility in Indian urban couples. J Reprod Infant Psychol 35: 353-364, 2017.

32. Lu Y, Yang L and Lu G: Mental status and personality of infertile women. Zhonghua Fu Chan Ke Za Zhi 30: 34-37, 1995 (In Chinese). 
33. Hunt $\mathbf{J}$ and Monach $\mathrm{JH}$ : Beyond bereavement model the significance of depression for infertile women. Fertil Steril 58: $1158-1163,1992$

34. Tarlatzis I, Tarlatzis BC, Diakogiannis I, Bontis J, Lagos S, Gavriilidou D and Mantalenakis S: Psychosocial impacts of infertility on Greek couples. Human Reprod 8: 396-401, 1993.

35. Conrad R, Schilling G, Langenbuch M, Haidl G and Liedtke R: Alexythymia in male infertility. Hum Reprod 16: 587-592, 2001.

36. Atherton F and Howel D: Psychological morbidity and the availability of assisted conception: A group comparison study. J Public Health Med 17: 157-160, 1995.

37. Berg BJ and Wilson JF: Psychological functioning across stages of treatment of infertility. J Behavior Med 14:11-26, 1991.

38. Whiteford LM and Gonzales L: Stigma: The hidden burden of infertility. Social Sci Med 40: 27-36, 1995.

39. Abbey A, Andrews FM and Halman LJ: Gender's role in responses to infertility. Psychol Women Q 15: 295-316, 1991.

40. Sezgin $\mathrm{H}$ and Hocaoglu C: Psychiatric aspects of infertility. Curr App Psychiatry 6: 165-184, 2014.

41. Nachtigall RD, Becker G and Wozny M: The effects of gender specific diagnosis on men's and women's response to infertility. Fertil Steril 57: 113-121, 1992.

42. Wischmann T, Stammer H, Scherg H, Gerhard I and Verres R Psychosocial characteristics of infertile couples: A study by the 'Heielberg fertility consultation service'. Human Reprod 16: 1753-1761, 2001.

43. Fassino S, Piero A, Boggio S, Piccioni V and Garzaro L: Anxiety, depression and anger suppression in infertile couples: A controlled study. Human Reprod 17: 2986-2994, 2002.

44. Yazdani F, Elyasi F, Peyvandi S, Moosazadeh M Galekolaee KS, Kalantari F, Rahmani Z and Hamzehgardeshi Z: Counseling-supportive interventions to decrease infertile women's perceived stress: A systematic review. Electron Physician 9: 4694-4702, 2017.
45. Zahra OA, Soheila R, Tahereh B, Marzieh A and Atefeh Y: The effectiveness of counseling with a cognitive-behavioral approach on infertile women's stress. Maedica (Bucur) 14: 363-370, 2019.

46. Mosalanejad L, Khodabakshi Koolaee A and Jamali S: Effect of cognitive behavioral therapy in mental health and hardiness of infertile women receiving assisted reproductive therapy (ART). Iran J Reprod Med 10: 483-488, 2012.

47. Maleki-Saghooni N, Amirian M, Sadeghi R and Latifnejad Roudsari R: Effectiveness of infertility counseling on pregnancy rate in infertile patients undergoing assisted reproductive technologies: A systematic review and meta-analysis. Int J Reprod Biomed 15: 391-402, 2017.

48. Burns LH: Psychiatric aspects of infertility and infertility treatments. Psychiatr Clin North Am 30: 689-716, 2007.

49. Dumbala S, Bhargav H, Satyanarayana V, Arasappa R. Varambally S, Desai G and Bangalore GN: Effect of yoga on psychological distress among women receiving treatment for infertility. Int J Yoga 13: 115-119, 2020.

50. Oron G, Allnutt E, Lackman T, Sokal-Arnon T, Holzer H and Takefman J: A prospective study using Hatha Yoga for stress reduction among women waiting for IVF treatment. Reprod Biomed Online 30: 542-548, 2015

51. Jasani S, Heller B, Jasulaitis S, Davidson M and Cytron JH: Impact of a structured yoga program on anxiety in infertility patients: A feasibility study. JFIV Reprod Med Genet 4: 2014-2016, 2016.

52. Hajishafiha M, Dehghan M, Kiarang N, Sadegh-Asadi N, Shayegh SN and Ghasemi-Rad M: Combined letrozole and clomiphene versus letrozole and clomiphene alone in infertile patients with polycystic ovary syndrome. Drug Des Devel Ther 7:1427-1431, 2013. 